\title{
Nuclear Magnetic Resonance Tomography
}

\author{
R.P. van Stapele and P.R. Locher, Eindhoven \\ (Philips' Research Laboratories)
}

\begin{abstract}
A summary of the lecture given to Council and invited participants on the occasion of the annual meeting held this year in Winterthur on 29 March. The principles of NMR tomography are discussed with particular emphasis on 2D and 3D Fourier imaging of protons.
\end{abstract}

The application of nuclear magnetic resonance to medical diagnosis was put forward by several researchers in 1970 and 1971. Of these, R. Damadian urged the matter strongly. He suggested pointby-point measurement of some characteristic NMR parameters in the human body, although this is very time-consuming. Shortly after, in 1973, P.C. Lauterbur proposed a much more efficient imaging method by which many points (e.g. a whole slice) are measured simultaneously.

Some university and industrial laboratories immediately became interested in the potential of the new imaging method and as early as 1980, at the IsmarAmpère Conference in Delft, W.C. Moore of the University of Nottingham could show impressive proton NMR images of the human head and lower parts of the human body.

In the first phase of development, which lasted about eight years, the proton with its high magnetic moment and high abundance was the only nucleus used for NMR imaging with magnetic fields up to about $0.15 \mathrm{~T}$, usually generated by a four coil resistive magnet. In the last four years, the strength of magnetic fields used for proton imaging has increased greatly. On a number of sites, which are not only in research institutes but also in some large hospitals, proton imaging is now done in large superconducting magnets with a field of $1.5 \mathrm{~T}$. Recently a so-called "whole body" superconducting magnet with a field of $2 \mathrm{~T}$ was installed at the Philips Forschungs Labor in Hamburg.

We shall discuss later whether such large magnetic fields are sensible tools for proton imaging. However, their availability makes imaging with nuclei other than protons possible. Moreover, it opens up the exciting field of NMR imaging combined with NMR spectroscopy.

In this article we discuss the principles of NMR imaging or NMR tomography, as it is now called, following closely the more extensive introductory article of P.R. Locher in Philips Technical Review, $41(1983 / 84)$ p. 73.

\section{Fundamentals of NMR}

Let us consider the protons in the liquid material found in most parts of the human body. The protons carry a small magnetic dipole moment. Due to thermal agitation, the total magnetic moment $m$ of the protons vanishes in the absence of a magnetic field. If we apply a magnetic field with strength $B_{0}$ to the human body a small magnetization $m_{0}$, proportional to $B_{0}$, is induced in the direction of the applied magnetic field. This magnetization can be brought into an excited state in which it makes an angle with the magnetic field resulting in the magnetization precessing about the field with the Larmor frequency $\omega=$ $\gamma B_{0}$, where $\gamma$ is the gyromagnetic ratio of the protons $(\gamma / 2 \pi=42.6 \mathrm{MHz} / \mathrm{T})$. Because of damping, the precessing magnetization slowly returns to the equilibrium state in such a way that its component $m_{\|}$, parallel with the magnetic field, increases quasi-exponentially with relaxation time $T_{1}$ to its equilibrium value $m_{o}$, whereas the component $m_{\perp}$, perpendicular to the magnetic field, vanishes quasi-exponentially with the often shorter relaxation time $T_{2}$.

Summarizing, we note that three parameters are important for NMR imaging of protons: the density $\rho$ of the protons, the parallel or spin-lattice relaxation time $T_{1}$ and the perpendicular relaxation time $T_{2}$. The different values that one or more of the three parameters have in different tissues determine the contrast in an NMR image. For protons in the human body, $T_{1}$ has values up to a few seconds, whereas $T_{2}$ ranges from milliseconds to seconds. The proton density changes with the water and fat content of the tissue.

\section{Imaging Equipment}

An NMR imaging apparatus or tomograph consists of a big magnet, gradient coils, radiofrequency (RF) coils, RF electronic equipment and a computer. The magnet should be large enough to enclose a human body and should produce a magnetic field with an inhomogeneity of less than $100 \mathrm{ppm}$ in a volume of say $40 \times 40 \times 30 \mathrm{~cm}$. The gradient coils should be able to produce three orthogonal gradients in the strength of the magnetic field with rise and decay times sufficiently short ( $\approx 1 \mathrm{~ms}$ ). The precession of the magnetization is detected by an RF coil, which has to fit round a human body. The RF equipment is the same as that of a standard NMR spec-

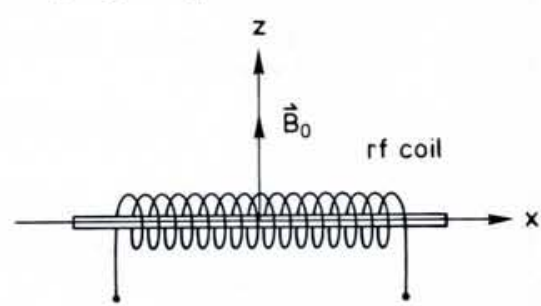

Fig. 1 - "One-dimensional" tube with a spatially-dependent proton density $\rho(x)$ in a homogeneous magnetic field $\mathrm{B}_{\mathrm{o}}$.

trometer. The computer system controls the RF pulses, the sequences of gradients applied and also computes the image from the voltage induced in the RF coil.

\section{Imaging of a 1D Object}

Let us place a one dimensional (1D) object with a non-uniform proton density $\rho(x)$ in a homogeneous magnetic field directed along the $z$-axis with strengths $B_{0}$ (see Fig. 1). We excite the proton spins with a strong short RF pulse with the RF magnetic field perpendicular to $B_{0}$. The angular frequency of the pulse $\omega$

Fig. 2 - a) Proton spin density as a function of $x$.

b) Total strength $B$ of the magnetic field with a gradient $G$ in the $x$-direction.

c) Signal spectrum of the precessing protons, which is an image of $\rho(x)$.
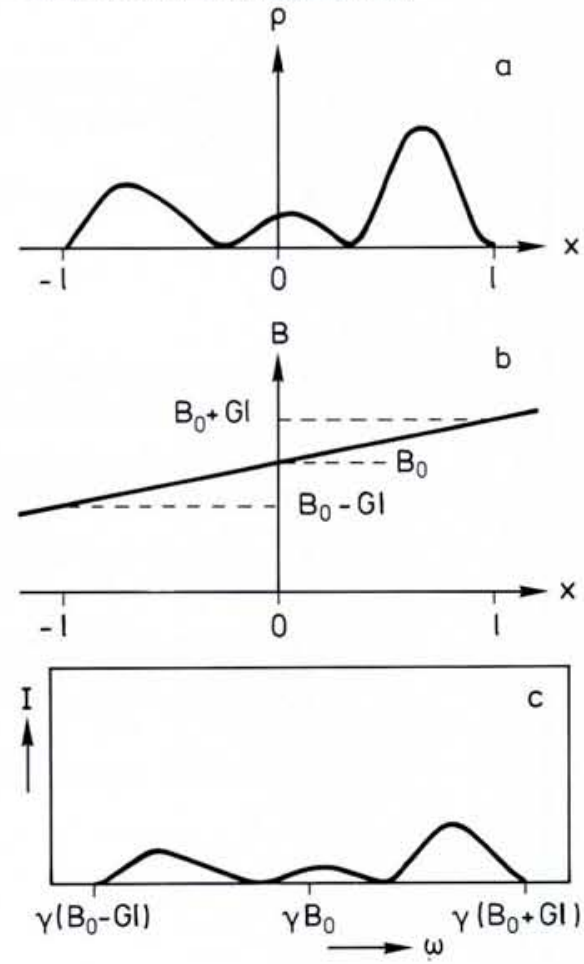
$=\gamma B_{0}$. During this RF pulse, the magnetization starts to precess about $\boldsymbol{B}_{0}$ in such a way that the angle between $\boldsymbol{B}$ 。 and the magnetization increases steadily. We end the pulse when the magnetization is precessing in the plane perpendicular to $B_{\mathrm{o}}$ (such an RF pulse is called a $90^{\circ}$-pulse). This completes the preparation stage.

After the pulse, at the detection stage of the measurement, we measure the voltage $V(t)$ induced in the RF coil, while we apply a gradient $G$ in the strength of the magnetic field. At point $x$ the field strength is $B(x)=B_{0}+G x$, so that the magnetization precesses with an angular frequency $\omega(x)=\gamma\left(B_{0}+G x\right)$. The spectrum of $V(t)$ induced by the precessing magnetization contains frequencies between $\gamma\left(B_{0}-G l\right)$ and $\gamma\left(B_{0}+G l\right)$ (see Fig. 2), where $2 /$ is the length of the object. The intensity $I(\omega)$ is proportional to the magnetization density of the protons at the point $\left[(\omega / \gamma)-B_{0}\right] / G$; in other words it is the image of the proton density. For the sake of simplicity we have neglected the decrease of the magnetization due to perpendicular relaxation during the detection of the induced signal. In practical circumstances, the image is determined both by the proton density and the relaxation times $T_{1}$ and $T_{2}$.

\section{Imaging of 2D and 3D Objects}

In order to extend the imaging method described in the previous section to $2 \mathrm{D}$ objects lying in the $x, y$-plane we need a gradient $G_{y}$ in the $y$-direction as well as a gradient $G_{x}$ in the $x$-direction. The gradient $G_{y}$ brings about an extra coding in the phases of the induced signals.

The various measuring methods are best discussed with the help of the formula for the voltage induced in the RF coil, as measured by double phase-sensitive detection:

$$
\begin{aligned}
S(t)= & \iint d x d y m_{\mathrm{L}}(x, y) \exp \mathrm{i} \gamma\left[x \int_{0}^{t} G_{x}(\tau) d \tau\right. \\
& \left.+y \int_{0}^{t} G_{y}(\tau] d \tau\right]
\end{aligned}
$$

This formula applies to the case where a two-dimensional object in the $x, y$ plane with a proton spin density $\rho(x, y)$ is excited by a suitable RF pulse. After this pulse, at $t=0$, the voltage induced in the $\mathrm{RF}$ coil by the precessing component $m_{\perp}(x, y)$ of the proton magnetization is measured, while two time-dependent gradients in the strength $B$ of the magnetic field $G_{x}=\partial \mathrm{B} / \partial \mathrm{x}$ and $G_{y}=$ $\partial B / \partial y$ are applied. This signal contains angular frequencies in a band with central frequenc $\gamma \omega_{0}=\gamma B_{0}$, where $B_{0}$ is the strength of the homogeneous magnetic field to which the gradient fields are added. Double phase-sensitive detection produces low-frequency signals.
We introduce a wave-vector:

$$
\boldsymbol{k}=\gamma \int_{1}^{t} \boldsymbol{G}(\tau) d \tau
$$

(the dimension of $\gamma G d \tau$ is $\mathrm{m}^{-1}$ ). Then, the function $S(t)$ corresponds to the function:

$$
F\left(k_{x}, k_{y}\right)=\iint_{\exp i\left(k_{x} x+k_{y} y\right)} d x d y m_{\mathrm{L}}(x, y)
$$

on the curve $\left(k_{x}(t), k_{y}(t)\right)$ determined by the gradients $G_{x}(t)$ and $G_{y}(t)$.

Let us assume that the function $F\left(k_{x^{\prime}}\right.$ $k_{y}$ ) has been determined experimentally from a great many measurements, each with different gradients $G_{x}(t)$ and/or $G_{y}(t)$. It is evident that $m_{\perp}(x, y)$ can be calculated from $F\left(k_{x}, k_{y}\right)$ by application of a two-dimensional Fourier transformation.

The density of perpendicular magnetization $m_{\perp}(x, y)$ is related to the proton density $\rho(x, y)$, the detailed relationship depending on the relaxation times $T_{1}$ and $T_{2}$, which in general vary with the position in the plane, and on the way in which the proton spins are excited.

In "2D Fourier imaging" the $k_{x^{\prime}}, k_{y}$ plane is filled with experimental data in a rectangular sequence. Constant gradients $G_{x}$ and $G_{y}$ are applied during a time $t_{y}$, followed by the measurement of the induced signal in the presence of a constant gradient $G_{x}$ only. This means that the induced signal is "plotted"

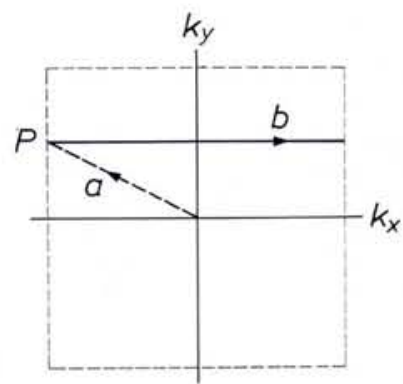

Fig. 3 -Excursion in the $k_{x}, k_{y}$-plane. Path a (evolution stage) is covered by making $G_{x}$ negative and (in the case illustrated) $G_{y}$ positive; the detection path $b$ is covered by making $G_{y}$ zero and $G_{x}$ positive.

along a line parallel to the $k_{x}$-axis at a distance

$$
k_{y}=\gamma \int_{0}^{t y} G_{y}(\tau) d \tau
$$

from the $k_{x}$-axis (see Fig. 3). In repeated measurements, the value of $k_{y}$ is changed by changing $G_{y}(\tau)$ and/or $t_{y}$. There are three stages in each measurement. The proton spins are excited at the preparation stage. The phases of the induced signal are coded during the evolution stage when the gradient $G_{y}$ is present. These phases do not depend on $x$. At the third stage, the detection stage, the spins precess in the presence of $G_{x}$ with an angular frequency depending on $x$ only. Between two measurements the magnetization should be allowed to

\section{QUEEN MARY COLLEGE University of London \\ NEW BLOOD LECTURESHIPS}

Applications are invited from those who will be under 35 on 1 October 1984 for posts established under the national scheme to encourage the appointment of young staff:

SCHOOL OF MATHEMATICAL SCIENCES - DYNAMICS: applicants should have made significant contributions to dynamics and its applications. The group's activities include the study of regular and chaotic motion in Hamiltonian and dissipative systems, and in a wide range of applications.

EXPERIMENTAL PARTICLE PHYSICS: OMC has an active and expanding programme: future experiments include electron-positron collisions at LEP, neutrino interactions at the SNS and nucleonnucleon polarization at LAMPF. There is a continuing programme at the proton-antiproton collider (UA1) and at LEAR (PS172). The Lecturer could join a current experiment and will be encouraged to take a major role in developing and exploiting the OPAL detector for LEP and in shaping the future programme of the group.

Salary scale (under review) : $£ 7190$ - $£ 14125$ p.a. plus $£ 1186$ London Allowance.

Application forms and further details obtainable from:

The Senior Personnel Officer, Queen Mary College,

Mile End Road, London E1 4NS (Telex 893750),

to be returned by 11 May.

Forms also available from Joyce Eggleton, CERN, or Peter Nichols, Rutherford Appleton Laboratory. Applicants resident abroad please send c.v. and list of publications and ask three referees to write directly to QMC. 

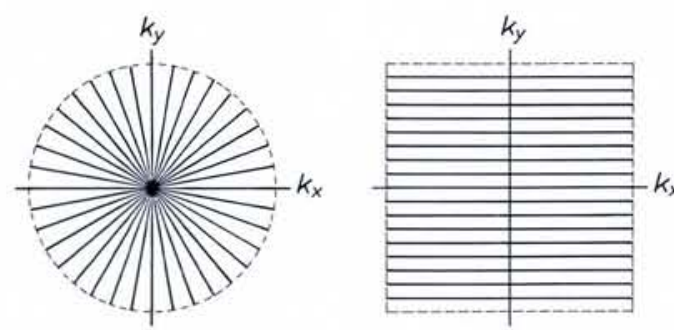

Fig. 4 - Projection-reconstruction method versus Fourier imaging. NMR signals are cross-sections of the function $F\left(k_{x}, k_{y}\right)$. The projection-reconstruction method detects the signals along lines through the origin in the $k_{x}, k_{y}$-plane (a), Fourier imaging detects the signals along parallel lines (b).

$\underline{a}$

$\underline{b}$

relax to its equilibrium state. This takes place in about $T$, seconds.

In practice a discrete Fourier transform is used. To obtain a $128 \times 128$ image we need values of $F\left(k_{x^{\prime}}, k_{y}\right.$ at 128 $\times 128$ points. This means that we have to make 128 measurements with different values of $k_{y}$, while we have to take 128 samples of each induced signal. With a waiting time of $T$, between two successive measurements we need about two minutes to complete the data acquisition for a $128 \times 128$ image.

An alternative method is known as "projection-reconstruction". This method uses a constant gradient in a direction $\alpha: G_{x}=G \cos \alpha$ and $G_{y}=G \sin \alpha$. The angle $\alpha$ is changed for successive measurements. By this procedure, the $k_{x}, k_{y}$-plane is filled with data in a radial

Fig. 5 - Practical example of a pulse and gradient sequence. The $90^{\circ}$ pulse at $P$ and the $180^{\circ}$ pulse at $Q$ give an echo signal with the centre at O.PR is the evolution stage, $R S$ $\left(=t_{w}\right)$ the detection time, $P O\left(=t_{e}\right.$ the echo time. The gradient $G_{z}$ is used in selecting a layer. The gradients $G_{x}$ and $G_{y}$ determine the path scanned in the $k_{x}, k_{y}$-plane.
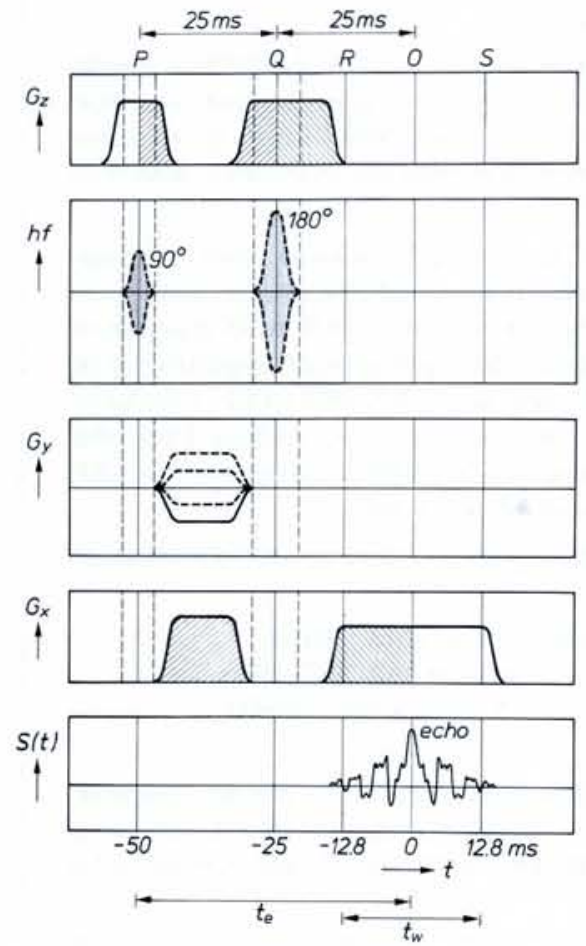

sequence (Fig. 4). In a single measurement, the induced signal is sampled along the line $k_{x}=\gamma G t \cos \alpha, k_{y}=\gamma G t$ $\sin \alpha$. The method is closely related to the imaging of $1 \mathrm{D}$ objects. In each single measurement, the induced signal with angular frequency $\omega$ results from the proton magnetization integrated along a line perpendicular to the gradient at a distance $\left[(\omega / \gamma)-B_{0}\right] / G$ from the origin. From this point of view you can consider that many one-dimensional projections of the two-dimensional object are measured. From these the magnetization density can be reconstructed.

The extension to imaging of three-dimensional objects is obvious. Before measuring the induced signals in the presence of a gradient, for example along the $x$-axis, we need to code the phase angles at the evolution stage using two orthogonal gradients, one along the $y$-axis and the other along the $z$-axis. By doing this, we fill the 3D $k$ space with induced signals, according to

$$
F(\boldsymbol{k})=\int_{\mathrm{vol}} d_{3} r m_{\perp}(\mathbf{r}) \exp \mathrm{i} \boldsymbol{k} \cdot \boldsymbol{r}
$$

The magnetization density is obtained as the 3D Fourier transform of $F(\boldsymbol{k})$.

We note that the data acquisition for a 3D image on a matrix of $n_{x} \cdot n_{y} \cdot n_{z}$ data points requires $n_{y} \cdot n_{z}$ separate measurements of induced signals. This makes 3D imaging time-consuming if we pursue a satisfactory resolution $\left(n_{y}=n_{z}=\right.$ 128 for example).

\section{Proton Imaging of the Human Body}

With the help of Figs. 5 and 6 we demonstrate the way in which an image of a slice of the human body is made by $2 \mathrm{D}$ Fourier imaging.

At the preparation stage, the slice to be measured is chosen by so-called "selective excitation". For simplicity we take a slice perpendicular to the $z$-axis. The $90^{\circ} \mathrm{RF}$ pulse is applied while a gradient $G_{z}$ in the strength of the magnetic field limits the resonance of the proton magnetization to the desired slice. For this purpose, the RF pulse must have a narrow spectral content. After the excitation, at the evolution stage, three gradients are applied. The gradients are designed to bring about the appropriate coding of the RF signal, which will be measured as an echo signal after a further RF pulse of $180^{\circ}$.

The gradient $G_{y}$ codes the phase angles. The gradient $G_{x}$ in whose presence the echo must be measured, is also applied at the evolution stage to ensure a perfect echo at the detection stage. The $180^{\circ}$ RF pulse, which is applied at a time $1 / 2 t_{\mathrm{e}}$ after the excitation pulse evokes an echo after a further $1 / 2 t_{\mathrm{e}}$. It is selectively applied to the selected slice with the help of a gradient $G_{z}$. The echo provides the induced signals which fill the $\left(k_{x^{\prime}}, k_{y}\right)$ plane. This measurement has to be repeated with different values of $G_{y}$. Between two successive measurements there is a waiting time of $t_{r}$ seconds in which the equilibrium of the proton magnetization is restored.

In a typical 2D Fourier imaging experiment with a slice $1 \mathrm{~cm}$ thick on a $128 \times$ 128 matrix in a magnetic field of $0.14 \mathrm{~T}$ we used $t_{\mathrm{e}}=50 \mathrm{~ms}$ and $t_{\mathrm{r}}=1 \mathrm{~s}$. With this repetition rate it takes about $2 \mathrm{~min}$ to acquire the data. The total measuring time can be shortened by decreasing $t_{r}$. Although the equilibrium is not then fully restored between the separate measurements, acceptable images are obtained. Experiments with $t_{r}=310 \mathrm{~ms}$ gave images of good quality in about $40 \mathrm{~s}$. As proposed by Van Uijen and Den Boef, the total measuring time can also be shortened if the equilibrium of the magnetization is restored by a driven-equilibrium technique. By this technique the combination of a $180^{\circ}$ RF pulse with a $90^{\circ}$ pulse rotates the magnetization back to the $z$-axis. Test experiments have shown that the method works, and moreover that the contrast between different kinds of tissue is strongly influenced. This makes the driven-equilibrium technique interesting as a method for contrast manipulation.

Fig. 6 - Path scanned in the $k_{x}, k_{y}$-plane with the pulse sequence of Fig. 5. The points $P, Q, R, O$ and $S$ correspond to those in Fig. 5 . The $180^{\circ}$ pulse corresponds to a reflection in the origin.

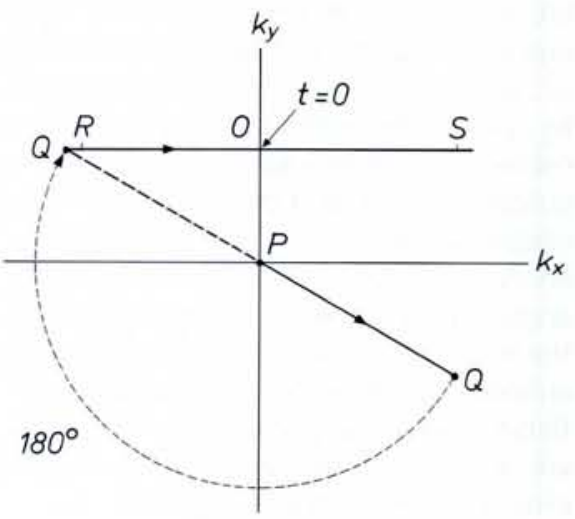


A different approach uses the fact that the induced signal is measured discretely. The time that elapses between two samples can be used to take samples from induced signals with different phase codings. This is accomplished by applying one steady gradient $G_{y}$ at the evolution stage (as in Fig. 5), whereas at the detection stage the constant gradient $G_{x}$ is combined with an oscillating gradient $G_{y}$. Samples of the induced signal are taken in such a way that data are measured simultaneously on a number of lines (say $n$ ) parallel to the $k_{x}$-axis. This shortens the total measuring time by a factor $n$. It should be noted that the signal-to-noise ratio is decreased by a factor $\sqrt{ } n$. This method, proposed by van Uijen, is intermediate between $2 \mathrm{D}$ Fourier imaging with constant gradients according to Kumar and Welti, and Ernst and Mansfield's echo-planar method in which the slice is imaged with a poor signal-to-noise ratio in one single measurement by means of an oscillating steep gradient that sweeps the vector $\boldsymbol{k}$ over the entire $k_{x^{\prime}}, k_{y}$-plane.

In medical applications it is often desirable to study a volume rather than a slice of the human body. A direct extension of $2 D$ to $3 D$ Fourier imaging would lead to an unacceptable total measuring time of $128 \times 128 \times 0.3 \mathrm{~s} \approx 82 \mathrm{~min}$ for an image on a $128 \times 128 \times 128$ matrix. Three methods, namely the multipleslice method of Crooks, "chunked" 3D Fourier imaging and fast Fourier imaging have proved to be feasible in reducing the measuring time sufficiently.

With the multiple-slice method, a number of parallel slices are measured simultaneously by $2 \mathrm{D}$ Fourier imaging. The time $t_{\mathrm{r}}$ between two single measurements of one slice is used to measure the other slices. With $t_{\mathrm{r}}=1 \mathrm{~s}$ it is possible to image 10 slices on a $128 \times 128$ matrix in $2 \mathrm{~min}$.

"Chunked" 3D Fourier imaging is 3D Fourier imaging with a limited resolution in one direction, say along the $z$-axis. After excitation of the proton magnetization and prior to data acquisition in the presence of the gradient $G_{x^{\prime}}$ the magnetization gains phase angles in the $G_{y}$ and $G_{z}$ gradients. For an image with a resolution of 15 points in the $z$-direction and 128 points in the $y$ and $x$-directions, $G_{z}$ and $G_{y}$ need $15 \times 128$ values, if 128 samples are to be taken from each induced signal. With a repetition time of $0.3 \mathrm{~s}$ an image of the 15 slices can be obtained in about $10 \mathrm{~min}$. When use is made of symmetries in the induced signals this can be reduced to about $5 \mathrm{~min}$.

We speak of fast Fourier imaging if the data are acquired simultaneously for a number of lines in the $\boldsymbol{k}$-space, as outlined above for 2D Fourier imaging. It is estimated that an image on a $128 \times 128$ $\times 128$ matrix with a reasonable signalto-noise ratio can be measured in less than ten minutes.

We end the discussion of proton imaging with some comments on the question of the optimal strength of the magnetic field. It can be shown that under certain assumptions (constant $Q$ ) the voltage signal-to-noise ratio increases in proportion with $B_{0}{ }^{3 / 2}$. This explains the tendency to use higher and higher field strengths during the past eight years. While in the beginning, resistive magnets operating at $0.1 \mathrm{~T}$ were used, superconducting magnets with fields as high as $2 \mathrm{~T}$ are now the fashion. The quality of the proton images has improved correspondingly, at least in fields up to $0.5 \mathrm{~T}$. As an example we show in Fig. $7 \mathrm{a}$ the proton image of a mid-sagittal slice of the human head and in Fig. 7b that of a sagittal slice of a female pelvis. Both images were made in a field of 0.5 T with the Gyroscan S5 system of Philips Medical Systems Division. Part of the improvement in quality is due to the better time stability of the field in superconducting magnets.

There are three reasons for expecting that the improvement in quality with higher fields will come to a halt. The first is that the penetration depth of the RF fields decreases with $\omega_{0}^{1 / 2}=\left(\gamma B_{0}\right)^{1 / 2}$. It eventually becomes so small that severe deviations in the phases and the magnitudes of the precessing perpendicular magnetization spoil the image. Although it was originally thought that difficulties would arise in fields of less than $1 \mathrm{~T}$, images obtained at 1.5 T do not show observable effects. The second reason is that it will be increasingly difficult to construct RF coils that fit the human body and also have an acceptable quality factor at the high frequencies of 60 to 85 $\mathrm{MHz}$. The third reason is that not all protons have the same resonance frequency at a given magnetic field strength. The frequency depends weakly on their chemical environment. So far we have neglected this so-called chemical shift, which amounts to some $10 \mathrm{ppm}$. At lower field strengths, the chemical shift does not influence the image. However, in fields of $1.5 \mathrm{~T}$ the chemical shift has been observed to give rise to ghost images which degrade the quality.

Although the chemical shift of the proton resonance frequency is a disadvantage for simple proton imaging in stronger fields, it opens new avenues for spatially-resolved NMR spectroscopy. If a Fourier imaging method is used so that
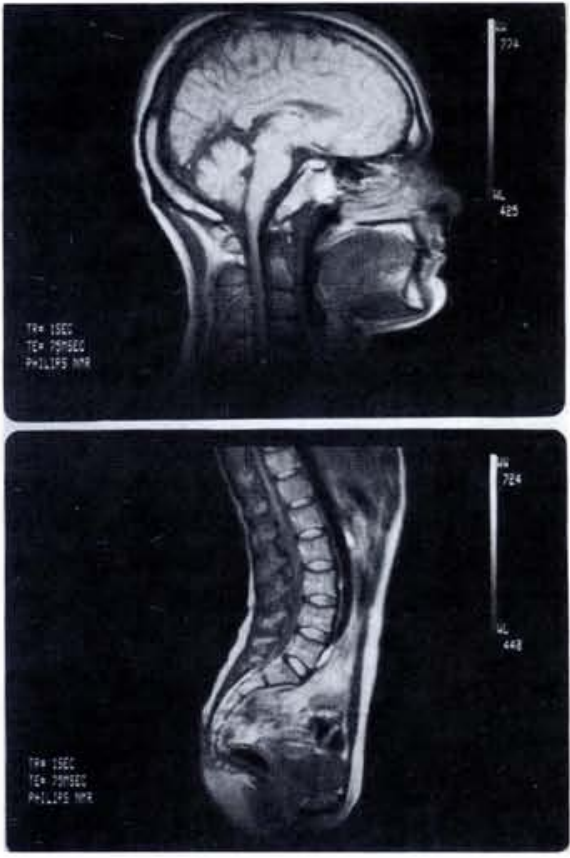

Fig. 7 - Proton images made in a magnetic field of $0.5 \mathrm{~T}$. The layers depicted have a thickness of $1 \mathrm{~cm}$.

the proton magnetization in a $2 \mathrm{D}$ slice gains phase angles in $G_{x}$ and $G_{y}$ gradients, whereupon the data are acquired in the absence of gradients, the 3D Fourier transform of the data gives an image of the spectrum of the protons on a chosen matrix in the $x, y$-plane. In animal experiments, spatially-resolved proton spectra have already been obtained. It is expected that future developments will make possible in vivo chemical analysis by proton NMR in the human body.

\section{Other Nuclei}

NMR tomography does not need to be restricted to protons. Although their NMR sensitivity and/or their abundance in the human body are less than those of protons, nuclei such as ${ }^{13} \mathrm{C},{ }^{23} \mathrm{Na},{ }^{31} \mathrm{P}$, ${ }^{19} \mathrm{~F}$ and ${ }^{14} \mathrm{~N}$ are in principle considered to be suitable for imaging or spatially-resolved NMR spectroscopy. However the experiments are at an early stage and the usefulness for medical diagnostics has still to be established. We mention one striking success scored recently by Hilal and co-workers at the Columbia Presbyterian Medical Center in New York. This group succeeded in making images of the sodium concentration in the living human brain. The images were made by 3D Fourier imaging at $1.5 \mathrm{~T}$ in a measuring time of 36 minutes. Images of sodium are important because the concentration of sodium that can be observed by NMR, rises in cells that are dying or dead. They can be used to determine the location of damage to the brain in stroke victims, for example. 